\title{
Noise-Enhanced Trapping in Chaotic Scattering
}

\author{
Eduardo G. Altmann ${ }^{1,2}$ and Antonio Endler ${ }^{1}$ \\ ${ }^{1}$ Instituto de Física, Universidade Federal do Rio Grande do Sul, 91501-970 Porto Alegre, Brazil \\ ${ }^{2}$ Max Planck Institute for the Physics of Complex Systems, 01187 Dresden, Germany
}

(Received 23 August 2010; published 9 December 2010)

\begin{abstract}
We show that noise enhances the trapping of trajectories in scattering systems. In fully chaotic systems, the decay rate can decrease with increasing noise due to a generic mismatch between the noiseless escape rate and the value predicted by the Liouville measure of the exit set. In Hamiltonian systems with mixed phase space we show that noise leads to a slower algebraic decay due to trajectories performing a random walk inside Kolmogorov-Arnold-Moser islands. We argue that these noise-enhanced trapping mechanisms exist in most scattering systems and are likely to be dominant for small noise intensities, which is confirmed through a detailed investigation in the Hénon map. Our results can be tested in fluid experiments, affect the fractal Weyl's law of quantum systems, and modify the estimations of chemical reaction rates based on phase-space transition state theory.
\end{abstract}

PACS numbers: 05.45.- a, 05.40.Ca, 05.40.Fb, 05.60.Cd

The responses of nonlinear dynamical systems to small uncorrelated random perturbations (noise) can be surprising and seemingly contradictory. Noise usually destroys fine structures of deterministic dynamics, e.g., it fattens fractals [1-3], but it can also combine constructively with the nonlinearities and increase the order of the system [4]. In chaotic Hamiltonian systems, investigations focused on the effect of noise on anomalous transport $[5,6]$ and, very recently, on chaotic scattering [7-9].

Chaotic scattering is a basic process of Hamiltonian dynamics $[2,10,11]$, with fundamental applications in classical [12] and quantum [2,13] systems, and recent applications ranging from plankton populations [3] to blood flows [14] and even the origin of life [15]. Chemical (dissociative) reactions of simple molecules are also scattering processes where chaos is essential in the microcanonical phase-space formulation of transition state theory (TST) [16]. Scattered trajectories perform transiently chaotic motion while trapped by fine structures of the phase space, such as fractal nonattracting sets and chains of Kolmogorov-Arnold-Moser (KAM) islands $[2,10]$. Noise destroys the small scales of these structures [7], modifies the temporal decay of trajectories in time from algebraic to exponential $[8,9]$ with an exponent that increases with noise [8], and creates otherwise forbidden escape paths [9]. All these effects weaken the deterministic trapping.

In this Letter we show that noise also plays a constructive role in chaotic scattering, enhancing the trapping of trajectories. First we introduce and scrutinize two different mechanisms responsible for this surprising effect, arguing that they exist in very general circumstances. The first mechanism acts in fully chaotic systems and reduces the escape rate of particles by blurring the natural measure of the system. The second mechanism acts on mixed-phasespace systems and enhances trapping by throwing trajectories inside KAM islands. We confirm the generality of these mechanisms through simulations in the conservative Hénon map, and we explore the implications of our results to physical systems.

To illustrate how noise enhances trapping in fully chaotic systems, consider the baker map [2]

$$
M \begin{cases}\left(x_{t+1}, y_{t+1}\right)=\left(x_{t} / 2,2 y_{t}\right) & y \leq 1 / 2 \\ \left(x_{t+1}, y_{t+1}\right)=\left(\left(x_{t}+1\right) / 2,2 y_{t}-1\right) & y>1 / 2,\end{cases}
$$

defined in $[0,1] \times[0,1]$. Escape is introduced through an arbitrary leak $I[17-20]$ :

$$
\tilde{M}\left(x_{t}, y_{t}\right)= \begin{cases}M\left(x_{t}, y_{t}\right) & \text { if }\left(x_{t}, y_{t}\right) \notin I \\ \text { escape } & \text { if }\left(x_{t}, y_{t}\right) \in I .\end{cases}
$$

For concreteness, consider $I$ to be a vertical stripe $(I=$ $\left.\left[x_{c}-\Delta_{x}, x_{c}+\Delta_{x}\right] \times[0,1]\right)$ at the center of the map $\left(x_{c}=\right.$ $\left.0.5, \Delta_{x}=0.05\right)$. The survival probability $P(t)$ of typical initial conditions decays asymptotically as $P(t) \sim e^{-t / \tau}$ $[2,10,11]$. In chemical reactions $P(t)$ corresponds to the reactant lifetime distribution, which plays a central role in TST [16]. The trapping strength is quantified through the characteristic lifetime $\tau$, which is the reciprocal of the escape rate and is different from the mean escape time [19]. It can be obtained from periodic orbits, e.g., by calculating the leading root $z^{\dagger}$ of the (truncated) polynomial approximation of the zeta function [10],

$$
1 / \zeta(z)=\prod_{p}\left(1-z^{t_{p}} / \Lambda_{p}\right), \quad \text { as } \tau_{\xi=0}=\ln z^{\dagger},
$$

where the product is taken over all periodic orbits $p$ which have period $t_{p}$ and expanding eigenvalue $\Lambda_{p}$, and $\xi=0$ indicates absence of noise [21]. We locate the orbits analytically so that Eq. (2) yields $\tau_{\xi=0}$ with a higher precision than simulations. For the baker map example above, all 1990 orbits up to period 18 yield $\tau_{\xi=0}=6.06 \pm 0.02$.

Next we consider the effect of noise, added independently to each trajectory $x \mapsto x+\xi \delta_{x}, y \mapsto y+\xi \delta_{y}$, 
where $\xi$ controls the noise intensity and $\delta_{x, y} \in[-1,1]$ are independent uniformly identically distributed stochastic variables. Let us first consider the simplest case of periodic boundary conditions (BC) for trajectories driven by noise outside $[0,1] \times[0,1]$. This would be the natural choice for maps defined on the torus. In this case, for $\xi \rightarrow \infty$ trajectories are uniformly distributed in $[0,1] \times[0,1]$ and $\tau \rightarrow$ $\tau^{*}$ with [17-19]

$$
\tau^{*}=\{-\ln [1-\mu(I)]\}^{-1}[\approx 1 / \mu(I) \text { for small } \mu(I)]
$$

where $\mu(I)$ is the Liouville measure (phase-space area) of the leak $I$. This is equivalent to the statistical microcanonical estimation of chemical reaction rates in TST [16]. In the baker's map example above, $\mu(I)=2 \Delta_{x}=0.1$ and $\tau^{*}=9.49$, which is greater than the noiseless case $\tau_{\xi=0}=$ 6.06 calculated above. The trapping is enhanced by noise.

Let us take a closer look at the two crucial points that lead to this simple yet surprising result. The first crucial point is the periodic BC that guarantees that $\tau \rightarrow \tau^{*}$ for $\xi \rightarrow \infty$. Another natural choice is open BC, in which case trajectories outside $[0,1] \times[0,1]$ escape. This type of escape prevails for large $\xi$ and reduces $\tau$ [for the baker map $\tau=-1 / \ln \left[\left(1-\Delta_{x}\right) /(2 \xi)^{2}\right] \sim 1 / \ln \xi$ for $\left.\xi \geq 1\right]$, but it is negligible for small $\xi$. The results shown in Fig. 1(a) confirm the existence of noise-enhanced trapping for open $\mathrm{BC}$, with a maximum $\tau_{m}>\tau_{\xi=0}$ at $\xi=\xi_{m}>0$. Noise increases $\tau$ by more than $50 \%$ in the periodic BC case and by almost $10 \%$ in the open $\mathrm{BC}$ case.

The second crucial point in the derivation above is $\tau^{*}>\tau_{\xi=0}$. References [17-20] show that this holds for most leaks, also for map (1) [18], which is confirmed in Fig. 1(b). For periodic BC the condition $\tau^{*}>\tau_{\xi=0}$ is sufficient for the existence of noise-enhanced trapping. For open BC the condition $\tau^{*}>\tau_{\xi=0}$ is necessary but not sufficient, and noise-enhanced trapping exists only when $\tau_{m}>\tau_{\xi=0}$. In Fig. 1(b) this is seen only in the deep
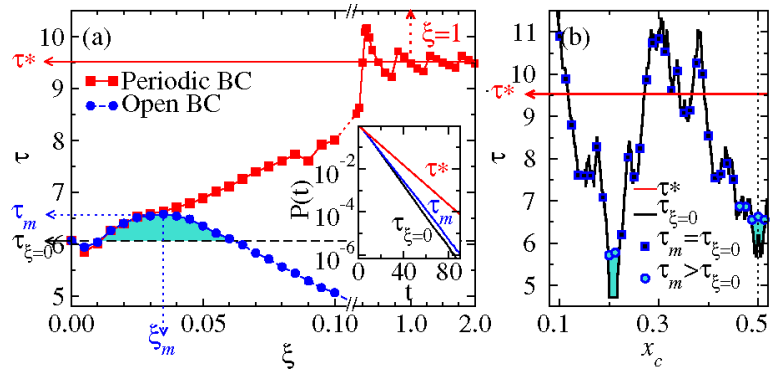

FIG. 1 (color online). Noise-enhanced trapping for fully chaotic systems. (a) The lifetime $\tau$ for the baker map (1) is shown as a function of the noise intensity $\xi$ for two BCs: periodic (red $\boldsymbol{\square}$ ) and open (blue 0 ). Horizontal lines: $\tau_{\xi=0}=6.06$ (solid) and $\tau^{*}=9.49$ (dashed). Inset: $P(t)$ for $\xi=0, \xi=\xi_{m}=0.035$ (open BC) and $\xi=1$ (periodic BC). (b) Values of $\tau^{*}, \tau_{\xi=0}$, and $\tau_{m}$ for different positions $x_{c}$ of the leak $\left(\Delta_{x}=0.05\right)$. Noiseenhanced trapping occurs when $\tau^{*}>\tau_{\xi=0}$ for periodic BC and when $\tau_{m}>\tau_{\xi=0}$ for open BC (blue shading). "valleys" of the $\tau_{\xi=0}\left(x_{c}\right)$ landscape. But the noiseenhanced mechanism is also present elsewhere; e.g., for several $x_{c}$ 's the $\tau(\xi)$ curve has local maxima. The difference between $\tau^{*}$ and $\tau_{\xi=0}$ is a consequence of the difference between the Liouville invariant density $\rho_{\mu}$ (uniform in $x, y$ ) and the open system's quasi-invariant density $\rho_{\xi=0}$ (nonuniform in $x, y$ ) inside $I[17,19]$. Note that escape occurs one iteration after trajectories enter $I$ (or leave $[0,1] \times[0,1])$. When $\rho_{\xi=0}$ is large in $I$, the open system measure of $I$ is larger than $\mu(I)$ and $\tau^{*}>\tau_{\xi=0}$; see Eq. (3). In this case the dominant effect of noise is to move trajectories outside $I$, avoiding their escape and increasing $\tau$. More generally, noise acts on $x, y$ coordinates making $\rho_{\xi}$ more uniform in $x, y[3,7]$, departing from $\rho_{\xi=0}$ and approaching $\rho_{\mu}$. This $\tau$-increasing effect is shown in Fig. 2 [17,22].

The mechanism described above acts on transient chaos and is different from stochastic and coherence resonance [4]. It has been observed in dissipative maps near crisis [24,25] and on extended excitable systems [26]. Strong nonlinearity was shown to be a sufficient condition for its occurrence in 1D maps [25]. No effect was observed in a 2D system [27], raising doubts about its generality in higher dimensions. Here we show the existence of this mechanism in (Hamiltonian) scattering systems. We identify $\tau^{*}>\tau_{\xi=0}$ as the crucial condition, clarifying the generality also for higher dimensions. A similar approach in 1D maps related this mechanism to the nonuniformity of the invariant density of the closed system [28]. In Hamiltonian systems the closed system density $\rho_{\mu}$ is uniform, but we show that the mechanism is nevertheless effective because the open system density $\rho_{\xi=0}$ is nonuniform.

In mixed-phase-space systems the exponential decay discussed so far is replaced by an algebraic decay $P(t) \sim$ $t^{-\alpha}$ due to the stickiness of KAM islands $[6,29]$. Figure 3
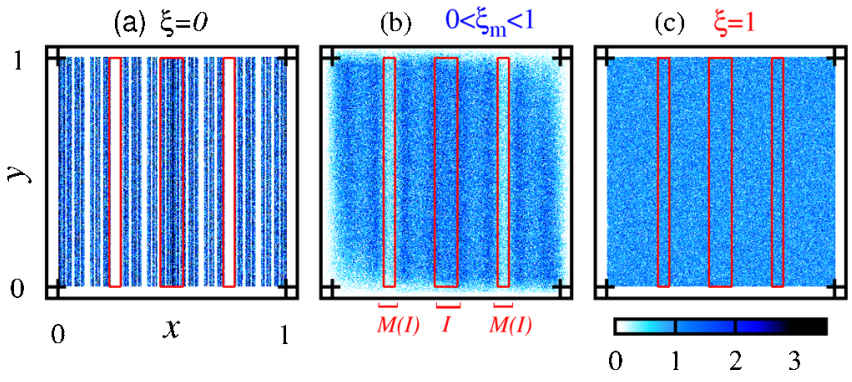

FIG. 2 (color online). Noise tends to uniformly distribute surviving trajectories. Quasi-invariant density $\rho_{\xi}$ of map (1) obtained at $t=40$ for (a) $\xi=0\left(\tau=\tau_{\xi=0}\right), \rho_{\xi=0}$ distributed along the unstable manifold of the chaotic saddle [2,10,11]; (b) $\xi=\xi_{m}\left(\tau=\tau_{m}\right.$, open BC), $\rho_{\xi}$ is smoothed in $I$ and negligible outside $[0,1] \times[0,1]$; (c) $\xi=1\left(\tau=\tau^{*}\right.$, periodic BC), $\rho_{\xi=1}=\rho_{\mu}$ is uniformly distributed. The leak $I\left(x_{c}=\right.$ $0.5, \Delta=0.05)$ and its forward iteration $M(I)$ are indicated. 

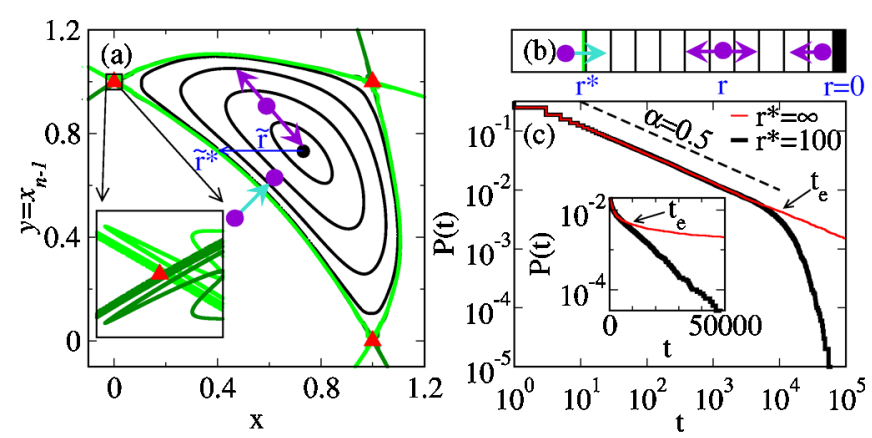

FIG. 3 (color online). Random-walk model for trajectories inside KAM islands. (a) Illustrative KAM island [Hénon map (4) with $k=2$ ]. Inset: Magnification around the period three orbit $\boldsymbol{\Delta}$ showing the intersection of manifolds. (b) Random-walk model with probabilities $p_{ \pm}=0.5$ of stepping $\Delta r= \pm 1$, one reflecting ( $r=0$, center of the island) and one absorbing boundary $\left(r=r^{*} \sim \tilde{r}^{*} / \xi\right.$, where $\tilde{r}^{*}$ is proportional to island width and $\xi$ to step length). (c) $P(t)$ for a simulation of $10^{5}$ walkers started in $r=r^{*}-1$, with $r^{*}=\infty$ (thin red line) and $r^{*}=100$ (thick black line). Dashed line: Scaling $\alpha=0.5$. Inset: Linear-log plot emphasizing the exponential tail (for $r^{*}=100$ ) starting at $t_{e} \approx$ $10^{4}=\left(r^{*}\right)^{2} \sim\left(\tilde{r}^{*} / \xi\right)^{2}$.

illustrates how noise enhances trapping in this case. If $\xi$ is larger than the lobes of manifold intersections close to the KAM island (see inset), it will be more likely for trajectories to approach (or enter) the region corresponding to the $\mathrm{KAM}$ island in the deterministic dynamics by jumping over the manifolds that shield the island. The time scales of this effect can be estimated following Ref. [5], which argues that for small $\xi$ the deterministic algebraic decay will be interrupted at a time $t_{b} \sim 1 / \xi^{\beta}$, where $\beta \approx 1$ depends on $\alpha$. An asymptotic exponential decay after such cutoff is the typical effect of noise in intermittent systems [5], observed also in scattering systems [8]. Our approach differs from Refs. [5,8] because we consider an additional trapping regime dominated by trajectories that jump inside the island, circle the elliptic fixed point, and perform a random walk in the perpendicular direction. This $1 \mathrm{D}$ random-walk model can be solved analytically in infinite domain [30] and yields $P(t) \sim t^{-\alpha}$ with $\alpha=0.5$. This is smaller than the deterministic exponent $1<\alpha_{\xi=0}<2$, meaning that trapping is enhanced. In reality, the island has a finite area that corresponds to a reflecting boundary in the model, as explained in Fig. 3(b). This introduces a cutoff $t_{e}>t_{b}$ in the $\alpha=0.5$ algebraic decay followed by an exponential decay, as shown in Fig. 3(c). This second cutoff occurs when trajectories explore the full island of size $\tilde{r}^{*}$, and since this is a diffusive process $\tilde{r}^{*}=r^{*} / \xi \sim \sqrt{t}$ and $t_{e} \sim 1 / \xi^{2}$ [6]. For $\xi \rightarrow 0$ the interval of enhanced trapping $\Delta t \equiv\left(t_{e}-t_{b}\right) \rightarrow \infty$ because of the different scalings of $t_{b}$ and $t_{e}$. Therefore, even if not valid asymptotically, enhanced trapping is in practice dominant for small $\xi$. Similar models have been used to investigate different problems: anomalous transport [6] and escape of initial conditions inside KAM islands in random maps [9].
Finally, to confirm the general validity of the two mechanisms discussed above, we investigate the paradigmatic Hénon map [31-33]

$$
x_{t+1}=k-x_{t}^{2}-x_{t-1}+\xi \delta_{t},
$$

where different random perturbations $\delta_{t}$, defined as before, are applied to each trajectory. To investigate the mechanism for fully chaotic systems we choose $k=6$ in Eq. (4), which is the smallest integer for which a complete horseshoe exists [33]. In Fig. 4(a) we identify in the phase space of map (4) the trapped region and exit set $I$ (reactant region and transition state in TST [16]). We estimate graphically [31] $\mu(I)=\operatorname{area}(I) /$ area(trapped region $)=0.2656$, which leads through Eq. (3) to $\tau^{*}=3.239$. We compute analytically [33] all 226 periodic orbits up to period 10, and through Eq. (2) we obtain $\tau_{\xi=0}=1.557 \pm 0.001$. Again $\tau^{*}>\tau_{\xi=0}$, fulfilling the necessary condition identified above for the existence of noise-enhanced trapping. Our numerical simulations reported in Fig. 4(b) confirm the nonmonotonic dependence of $\tau$ on $\xi$ with $\tau_{m}>\tau_{\xi=0}$, in perfect analogy with the results for the baker map shown in Fig. 1. To investigate the mechanism for mixed-phasespace systems we choose $k=2$ in Eq. (4). The phase space is similar to the one shown in Fig. 4(a) except for the KAM island in Fig. 3(a) around the fixed point (black dot ). The results are summarized in Fig. 5 and confirm quantitatively the predictions (scalings of $t_{b, e}$ and $\alpha=0.5$ ) of the random-walk model in Figs. 3(b) and 3(c).

In summary, we have shown that weak noise leads to a slower decay of the survival probability $P(t)$ in fully chaotic and in mixed-phase-space scattering systems. In both cases $P(t)$ changes nonmonotonically with noise intensity $\xi$ (Figs. 4 and 5), an effect previously observed in the dependence of the diffusion coefficient on $\xi[6,34]$. Our
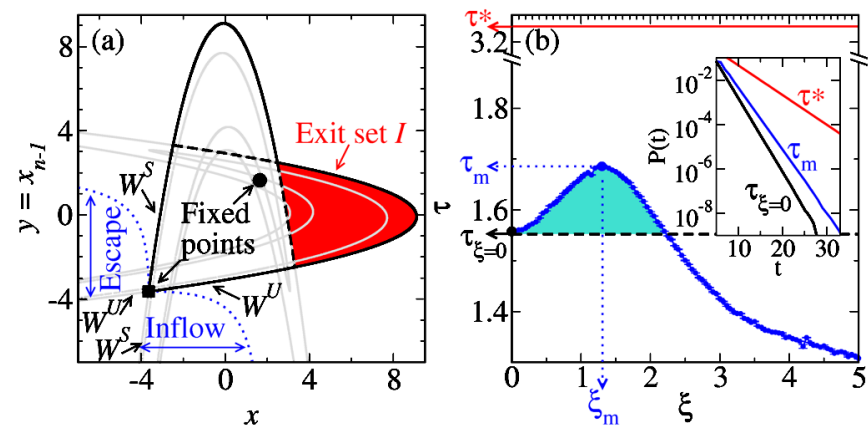

FIG. 4 (color online). Noise-enhanced trapping in the fully chaotic $(k=6)$ Hénon map (4). (a) Phase space with trapped region, delimited by the solid black line (first intersection of the manifolds $W^{U, S}$ of the fixed point $\square$ ). Escape (inflow) denotes an unbounded forward (backward) invariant set that diverges in forward (backward) iterations [32]. Initial conditions and the region where trajectories are removed were chosen inside these sets [35]. The exit set $I$ is the set of points that exit the trapped region in one iteration [31]. (b) Dependence of $\tau$ on $\xi$ with $\tau_{\xi=0}=1.557$ (dashed line) and $\tau^{*}=3.239$ (dotted line). Inset: $P(t)$ for $\xi=0, \xi=\xi_{m}$, and with $\tau^{*}$. 


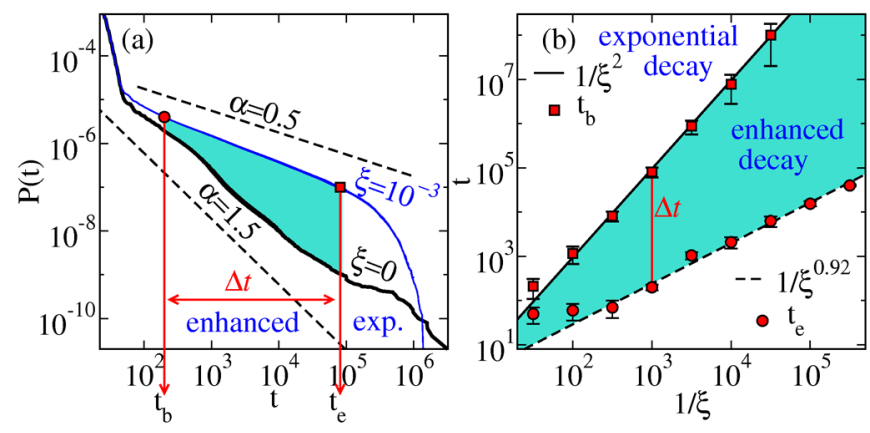

FIG. 5 (color online). Noise-enhanced trapping in the Hénon map (4) with mixed phase space $(k=2)$. (a) $P(t)$ for $\xi=0$ (thick black line) and $\xi=10^{-3}$ (thin blue line) [35]. The times $t_{b}$ and $t_{e}$ indicate the beginning and end of the mechanism described in Fig. 3. Power-law scalings are shown as a reference (dashed lines). (b) Dependence of $t_{b}$ and $t_{e}$ on $\xi$ with fits of the model predictions (with $\beta=0.92$; see text).

approach to the mechanism for fully chaotic systems extends naturally to higher dimensions, while the mixedphase-space mechanism has to be expanded to take Arnold's diffusion into account [2].

All scattering systems are likely to be subject to small noiselike perturbations and to experience an enhancement of the trapping. Experiments on the chaotic advection of passive tracers [12] can provide a direct test of our predictions: by systematically changing the properties of the tracer and fluid one can control the molecular diffusion $\left(\sim \xi^{2}\right)$ and obtain the dependence of $P(t)$ on $\xi$.

Our results also describe the effect of noise in the phasespace formulation of TST, where an increase of the chemical reaction rate is expected. Simulations of unimolecular reactions with mixed phase space found $P(t) \sim t^{-\alpha}$ with $\alpha<1$ and an exponential tail. According to Ref. [16] the dynamical origins of this behavior remain obscure. We show that noise leads to the same observations. It remains to be shown whether this explains the previous simulations, where noiselike perturbations could originate from roundoff errors or from higher degrees of freedom [6]. Our results also impact quantum systems. For instance, the increase in the characteristic lifetime $\tau$ indicates a modification of the fractal dimensions of the invariant sets $[3,22,23]$ and consequently of the fractal Weyl's law [13].

We are indebted to T. Tél and D. Pazó for insightful suggestions. E. G. A. was supported by the Max Planck Society.

[1] A. Ben-Mizrachi, I. Procaccia, and P. Grassberger, Phys. Rev. A 29, 975 (1984).

[2] E. Ott, Chaos In Dynamical Systems (Cambridge University Press, Cambridge, England, 2002).

[3] T. Tél et al., Chaos 10, 89 (2000).

[4] See, e.g., A. S. Pikovsky and J. Kurths, Phys. Rev. Lett. 78, 775 (1997); L. Gammaitoni, P. Hänggi, P. Jung, and F. Marchesoni, Rev. Mod. Phys. 70, 223 (1998).
[5] E. Floriani, R. Mannella, and P. Grigolini, Phys. Rev. E 52, 5910 (1995).

[6] E. G. Altmann and H. Kantz, Europhys. Lett. 78, 10008 (2007); in Anomalous Transport: Foundations and Applications, edited by R. Klages, G. Radons, and I. M. Sokolov (Wiley-VCH, Weinheim, 2008), p. 271; E. G. Altmann, Ph.D. thesis, Wuppertal University, 2007.

[7] P. Mills, Commun. Nonlinear Sci. Numer. Simul. 11, 899 (2006).

[8] J. M. Seoane and M. A. F. Sanjuán, Phys. Lett. A 372, 110 (2007); J. M. Seoane, L. Huang, M. A. F. Sanjuán, and Y.-C. Lai, Phys. Rev. E 79, 047202 (2009).

[9] C.S. Rodrigues, A.P.S. de Moura, and C. Grebogi, Phys. Rev. E 82, 026211 (2010).

[10] P. Gaspard, Chaos, Scattering And Statistical Mechanics (Cambridge University Press, Cambridge, England, 1998).

[11] T. Tél, and M. Gruiz, Chaotic Dynamics (Cambridge University Press, Cambridge, England, 2006).

[12] J. C. Sommerer, H. C. Ku, and H. E. Gilreath, Phys. Rev. Lett. 77, 5055 (1996).

[13] W. T. Lu, S. Sridhar, and M. Zworski, Phys. Rev. Lett. 91, 154101 (2003).

[14] A. B. Schelin et al., Phys. Rev. E 80, 016213 (2009).

[15] I. Scheuring, T. Czárán, P. Szabó, G. Károlyi, and Z. Toroczkai, Origins Life Evol. Biosphere 33, 319 (2003).

[16] See G. S. Ezra, H. Waalkens, and S. Wiggins, J. Chem. Phys. 130, 164118 (2009), and references therein.

[17] V. Paar and N. Pavin, Phys. Rev. E 55, 4112 (1997); V. Paar and H. Buljan, ibid. 62, 4869 (2000).

[18] J. Schneider, T. Tél, and Z. Neufeld, Phys. Rev. E 66, 066218 (2002).

[19] E. G. Altmann and T. Tél, Phys. Rev. Lett. 100, 174101 (2008); Phys. Rev. E 79, 016204 (2009).

[20] V. S. Afraimovich and L. A. Bunimovich, Nonlinearity 23, 643 (2010); L.A. Bunimovich and A. Yurchenko, arXiv:0811.4438.

[21] For noise corrections, see C. P. Dettmann, R. Mainieri, and G. Vattay, J. Stat. Phys. 93, 981 (1998).

[22] We have verified that trapping is enhanced also for random maps, where dimensions remain fractal $[3,23]$.

[23] F. J. Romeiras, C. Grebogi, and E. Ott, Phys. Rev. A 41, 784 (1990).

[24] M. Franaszek, Phys. Rev. A 44, 4065 (1991).

[25] P. Reimann, J. Stat. Phys. 85, 403 (1996).

[26] R. Wackerbauer and S. Kobayashi, Phys. Rev. E 75, 066209 (2007).

[27] J. A. Blackburn, N. Gronbech-Jensen, and H. J. T. Smith, Phys. Rev. Lett. 74, 908 (1995).

[28] H. Faisst and B. Eckhardt, Phys. Rev. E 68, 026215 (2003).

[29] G. M. Zaslavsky, Phys. Rep. 371, 461 (2002).

[30] W. Feller, An Introduction To Probability Theory And Its Applications (John Wiley \& Sons, New York, 1950).

[31] J. Meiss, Chaos 7, 139 (1997).

[32] E. Petrisor, Chaos Solitons Fractals 17, 651 (2003).

[33] A. Endler and J.A.C. Gallas, Phys. Lett. A 352, 124 (2006); 356, 1 (2006).

[34] R. Klages, Europhys. Lett. 57, 796 (2002).

[35] Trajectories were started at $x_{i} \in\left\{0.9 x_{f p}, 1.0 x_{f p}\right\}, y_{i}=$ $1.1 y_{f p}$ and removed at $x<1.1 x_{f p}, y<1.1 y_{f p}$, where $x_{f p}=y_{f p}=-1-\sqrt{1+k}[31-33]$. 